\title{
Norois
}

Environnement, aménagement, société

\section{Utilisation de données SPOT5 pour la cartographie des habitats benthiques littoraux. Application à l'archipel des îles Chausey (golfe normand-breton, France)}

First assessment of SPOT5 ability to map coastal benthic habitats: application to the Chausey Islands, English Channel

Adeline Cotonnec, Pascal Gouéry, Mouna Mokrani, Jérôme Fournier, Brice Anselme, Alain Dréau, Vincent Dubreuil, Andrea C. Panizza et Pascal Talec

\section{OpenEdition}

Journals

Édition électronique

URL : https://journals.openedition.org/norois/393

DOI : $10.4000 /$ norois.393

ISBN : 978-2-7535-1544-4

ISSN : 1760-8546

Éditeur

Presses universitaires de Rennes

Édition imprimée

Date de publication : 1 septembre 2005

Pagination : $37-50$

ISBN : 978-2-7535-0218-5

ISSN : 0029-182X

Référence électronique

Adeline Cotonnec, Pascal Gouéry, Mouna Mokrani, Jérôme Fournier, Brice Anselme, Alain Dréau, Vincent Dubreuil, Andrea C. Panizza et Pascal Talec, « Utilisation de données SPOT5 pour la cartographie des habitats benthiques littoraux. Application à l'archipel des îles Chausey (golfe normand-breton, France) », Norois [En ligne], 196 | 2005/3, mis en ligne le 15 décembre 2008, consulté le 13 janvier 2022. URL : http://journals.openedition.org/norois/393 ; DOI : https://doi.org/10.4000/ norois.393

Ce document a été généré automatiquement le 13 janvier 2022.

(c) Tous droits réservés 


\section{Utilisation de données SPOT5 pour la cartographie des habitats benthiques littoraux. Application à l'archipel des îles Chausey (golfe normand-breton, France)}

First assessment of SPOT5 ability to map coastal benthic habitats: application to the Chausey Islands, English Channel

Adeline Cotonnec, Pascal Gouéry, Mouna Mokrani, Jérôme Fournier, Brice Anselme, Alain Dréau, Vincent Dubreuil, Andrea C. Panizza et Pascal Talec

\section{NOTE DE L'ÉDITEUR}

Cet article a été reçu le 20 mai 2005 et définitivement accepté le 25 octobre 2005.

1 L'archipel des îles Chausey (golfe normand-breton) fait actuellement l'objet d'importants conflits d'usages essentiellement générés par une fréquentation croissante des îles et de l'estran qui se traduit sur les composantes « naturelles » de ses paysages. Certains de ces habitats font aujourd'hui l'objet d'un recensement dans le cadre de la directive européenne «Habitats» (directive 92/93 CEE du 21 mai 1992). La mise en œuvre de cette directive dont l'objectif est la préservation des habitats naturels passe d'une part, par un état des lieux de ces habitats et d'autre part, par la mise en place tous les 6 ans d'un suivi permettant de caractériser le bon état de conservation des milieux inventoriés. Ainsi, pour répondre aux impératifs d'inventaires, de gestion et de protection du territoire sur l'archipel, les gestionnaires ${ }^{1}$ doivent avoir recours à des documents cartographiques fiables et récents. Or, la complexité de ce milieu rend difficile la réalisation de tels documents car elle se heurte à la disponibilité des moyens d'acquisition et à la mise en œuvre de techniques de traitement des données adaptées à 
la variabilité spatiale et temporelle des habitats qui constituent le paysage si caractéristique de cet archipel. L'identification et le suivi des composantes du milieu naturel (couverture algale, herbiers de phanérogames, bancs de sable, etc.) requièrent des acquisitions à grande échelle $(<1 / 25000)$ et/ou des résolutions spatiales fines $(<5$ m) (Fournier, 2003).

Dans ce contexte, il est intéressant d'évaluer les potentialités des images à haute résolution de SPOT5 pour l'identification des habitats naturels répondant à la codification «NATURA 2000 ». L'objectif de ce travail réside ainsi dans l'apport d'éléments de réponses méthodologiques et techniques à la problématique de discrimination et d'inventaire des éléments du paysage littoral, en mettant l'accent sur les principales difficultés rencontrées dans ce domaine. Ces premiers résultats s'inscrivent dans les problématiques émanant de l'appel à proposition du CNES et de l'IFEN concernant le suivi du littoral par le satellite SPOT5 (Fournier et al., 2004).

\section{Présentation du site}

3 L'archipel des îles Chausey est situé au cœur du golfe normand-breton, il est rattaché administrativement à la commune de Granville (Basse-Normandie) (fig. 1). Il compte 52 îlots toujours émergés, dont 38 sont végétalisés et 14 de simples rochers. L'estran s'étend d'est en ouest sur $12 \mathrm{~km}$ et du nord au sud sur 5,5 km. Les îles et l'estran représentent environ 5000 hectares. La moitié de l'archipel se situe ainsi en dessous du 0 marégraphique (SHOM). L'espace terrestre ne représente que 2,5\% de l'ensemble de l'archipel.

Figure 1 : Localisation de l'archipel des îles Chausey

\section{Location of the Chausey Islands study area}

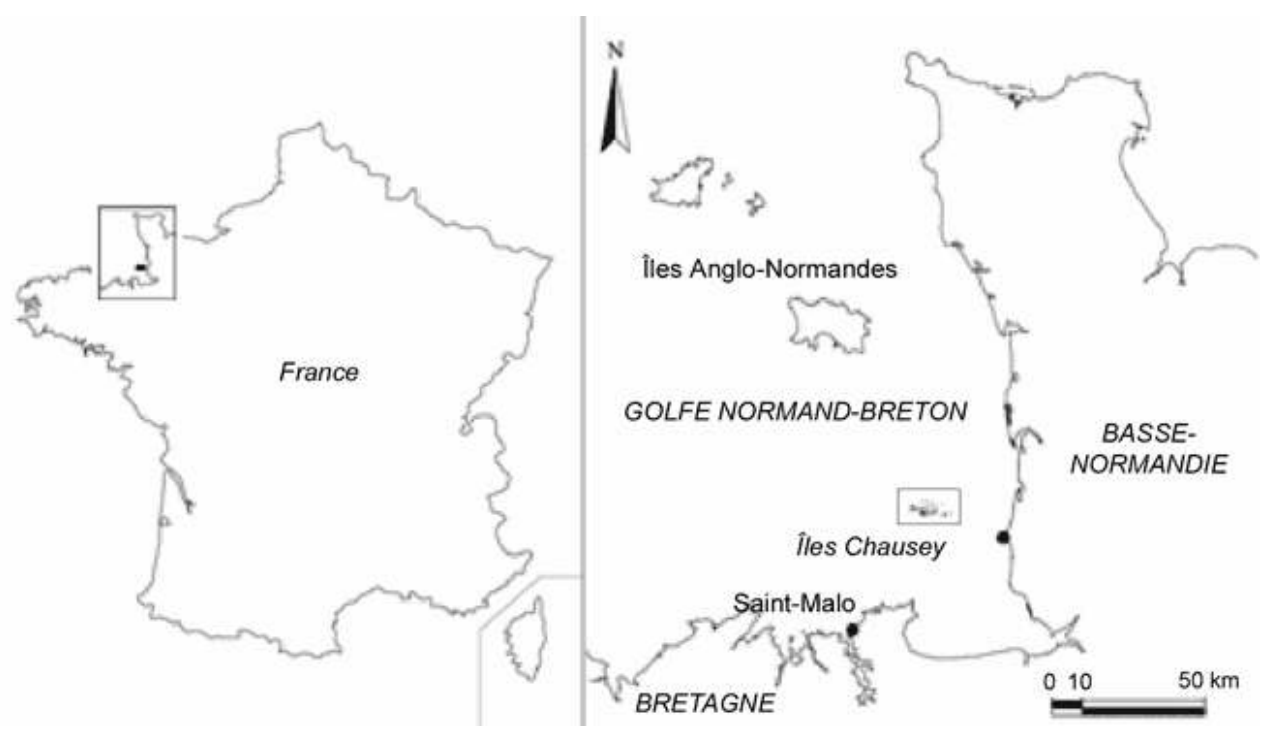

L'intérêt du site réside dans son régime hydrodynamique. En effet, ce complexe d'îles se situe dans une mer à régime mégatidal où le marnage atteint, par grands coefficients, près de 14 mètres. En raison de ce très fort marnage, les courants particulièrement puissants modèlent profondément l'estran par la création de figures sédimentaires 
extrêmement variées (ripples-marks, structures linguoïdales, ridins, rides et mégarides, langues sableuses, etc.). Ils contribuent, dans la partie orientale plus exposée, à l'existence de vastes bancs sableux, fins à grossiers, souvent bioclastiques. À l'inverse, on observe dans la partie centrale et surtout dans le secteur occidental, plus protégés des houles et des courants par la multitude d'îlots, des faciès de sédiments plus vaseux.

Les caractéristiques hydrodynamique et morphologique expliquent en grande partie la diversité des paysages et des habitats présents dans l'archipel. Sur l'estran, les habitats benthiques se juxtaposent en une mosaïque spatiale particulièrement complexe. L'étagement qui résulte de ces facteurs est constitué par des ceintures plus ou moins continues (Fournier et Le Vot, 2004). L'étage infralittoral est essentiellement occupé par les laminariales et les herbiers de phanérogame marine (Zostera marina). Dans la zone de balancement des marées qui correspond à l'étage médiolittoral se succèdent différentes ceintures de fucales: Fucus serratus en mode battu, Ascophyllum nodosum, Fucus vesiculosus et Fucus spiralis en mode abrité, ainsi que Pelvetia canaliculata qui marque la limite supérieure des marées de morte eau. Les faciès sableux sont essentiellement colonisés par un polychète (Lanice conchilega) et une phanérogame marine (Zostera noltii) ; les faciès vaseux par un fucus vasicole (Fucus lutarius) et par la vauchérie (Vaucheria dichotoma var. marina). L'étage supralittoral présente les ceintures classiques à lichens. On distingue la première bande sombre caractéristique colonisée par Verrucaria maura, puis une seconde jaune-orangée colonisée par Xanthoria parietina et Caloplaca marina. Cet étage est limité dans sa partie supérieure par les premières plantes halo-tolérantes, notamment Armeria maritima et Crithmum maritimum.

\section{Matériel et méthodes}

\section{Disponibilité des données et prétraitements}

6 Depuis près de 40 années, le golfe normand-breton fait l'objet d'études approfondies (Bonnot-Courtois et al., 2002). Une base conséquente de données géographiques est aujourd'hui constituée sur l'archipel de Chausey. Ces études concernent l'écologie et la biogéographie des principales biocénoses benthiques et s'appuient sur des travaux de terrain et de cartographies des habitats benthiques réalisées à l'aide de photographies aériennes récentes (Belsher, 1985 ; Bonnot-Courtois et al., sous presse ; Dréau, 2003). De nombreux travaux ont porté sur le traitement des images satellitales pour la cartographie des littoraux (Belsher, 1990; Ben Moussa et al., 1989; Chauvaud et al., 2001 ; Loubersac et Belbeoch, 1984 ; Meinesz et al., 1991). La précision spatiale et la discrimination des habitats étaient toutefois encore assez décevantes bien que des avancées conséquentes aient été réalisées (Andréfouët et al., 2001 ; Guillaumont et al., 1993). Depuis quelques années, la résolution spatiale des images issues des capteurs récents s'est nettement améliorée et permet de cartographier les littoraux plus précisément (Guillaumont et al., 1997). Plusieurs capteurs sont actuellement utilisés :

- Les capteurs aéroportés sont nettement supérieurs en matière de résolution spatiale mais restent toutefois difficiles à utiliser en raison de la logistique lourde à mettre en place et du coût parfois prohibitif des études (Dierssen et al., 2003 ; Pasqualini et al., 1997 ; Populus et al., 2004 ; Shanmugan et al., 2003).

- Les capteurs satellitaires utilisés pour la cartographie des estrans sont essentiellement IKONOS (Andréfouët et al., 2003 ; Mumby et Edwards, 2002) qui offre une résolution spatiale 
proche du mètre et SPOT5 (Fournier et al., 2004 ; Mokrani, 2004 ; Pasqualini et al., 2005) dont

l'amélioration de la résolution spatiale offre de nouvelles perspectives d'utilisation.

La disponibilité des images visant à mettre en évidence les habitats benthiques de l'archipel des îles Chausey dépend de contraintes inhérentes aux dates de passage des capteurs. La fréquence de passage des satellites SPOT permet d'envisager une situation théorique optimale de plusieurs images par an. Or, la disponibilité des images est soumise aux situations météorologiques, souvent défavorables sur le golfe normandbreton, et à de fortes contraintes marégraphiques nécessitant une programmation auprès du CNES. Ainsi, pour cette étude, une scène SPOT5 centrée sur l'archipel et acquise le 14 mai 2003 à $11 \mathrm{~h} 33$ a été utilisée. Le choix des images s'est porté sur des données multispectrales $(0,50-0,59 \mu \mathrm{m} ; 0,61-0,68 \mu \mathrm{m}$ et $0,78-0,89 \mu \mathrm{m})$ à $2,5 \mathrm{~m}$ de résolution (THR + XS).

Des photographies aériennes ont été utilisées comme documents de référence et de validation. La mission DIREN/IGN du 13 août 2002 au 1/10 000 couleur a été retenue car cette saison est plus propice à l'observation - et donc à la détection - des herbiers à Zostères qui présentent alors un développement foliaire maximal. De plus, cette mission permet une couverture complète et stéréoscopique de l'archipel (fig. 2). Les photographies infrarouges ne sont pas exploitées ici car l'absorption très forte des longueurs d'onde par l'eau dans le proche infrarouge perturbe considérablement la détection de la flore immergée.

Figure 2 : Localisation des missions terrain sur l'archipel des Chausey - Mission aérienne DIREN/ IGN 2002

Localisation of field mission, Chausey archipelago

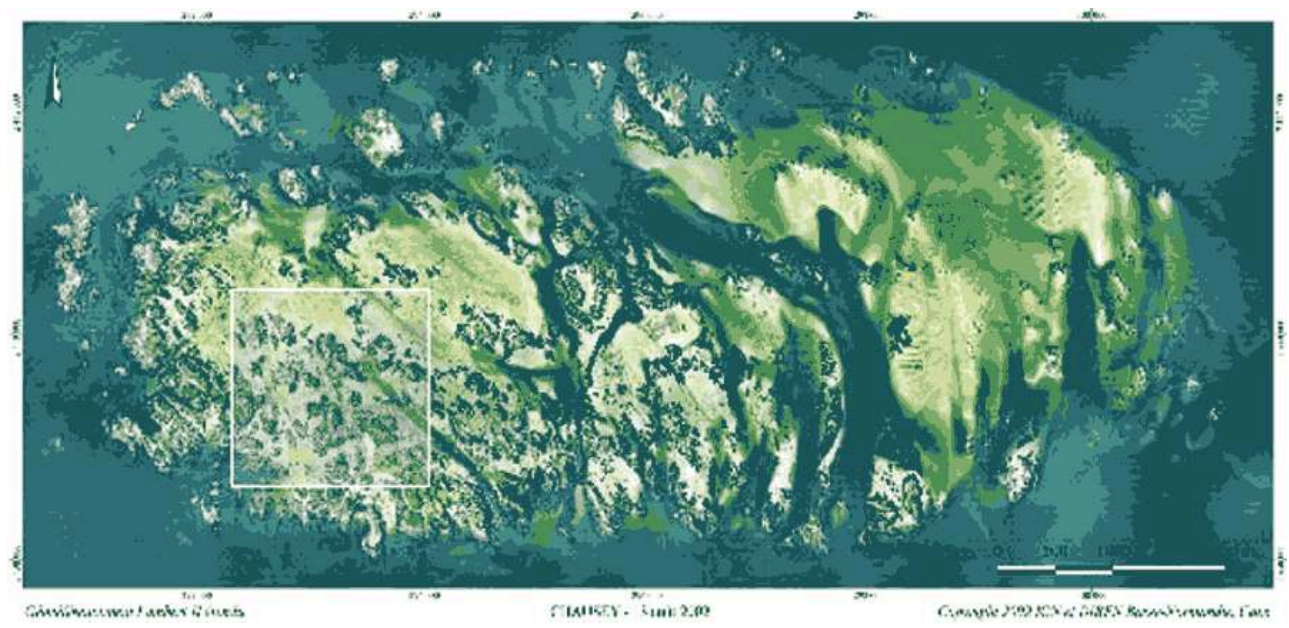

9 La qualité des images SPOT5 s'apprécie à travers l'analyse d'une composition colorée avec l'attribution des couleurs rouge, vert et bleu respectivement aux canaux XS3, XS2 et XS1 (fig. 3). Elle permet de vérifier visuellement la couverture de la scène et d'identifier la présence des principaux objets de l'archipel (îles et îlots, estran). 
Figure 3 : Composition colorée Xs3 (R) Xs2 (V) Xs1 (B), Image SPOT5 du 14 mai 2003-archipel des Chausey

Spot5 image (bands 3-red, 2-green, and 1-blue) of Chausey Islands (14-03-05)

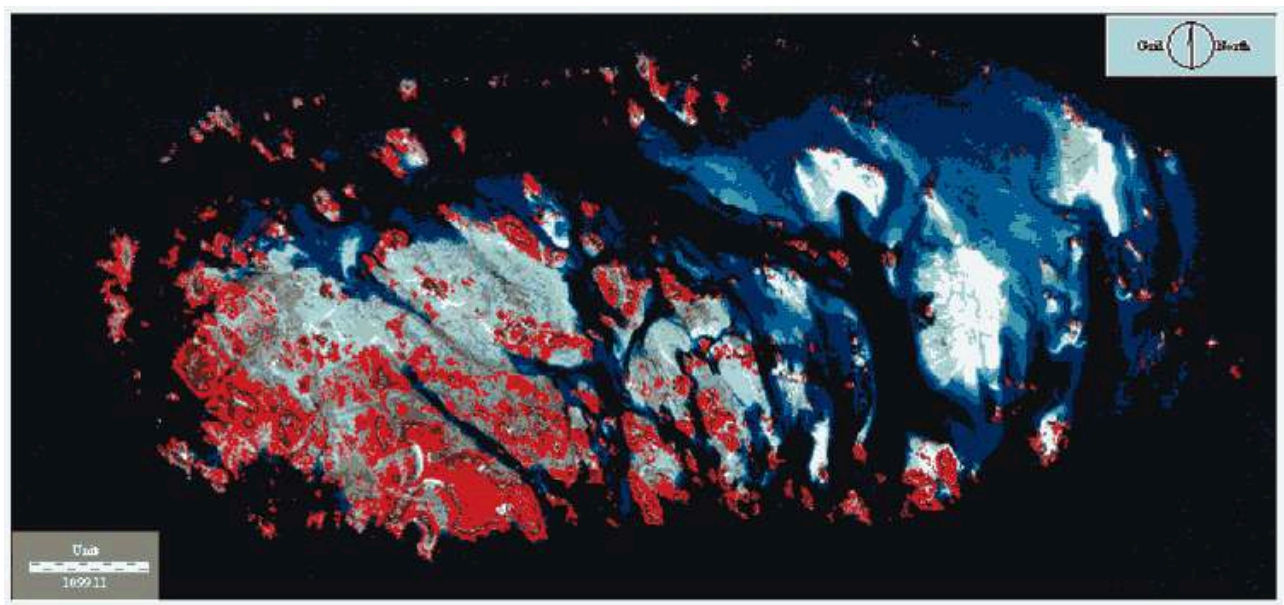

10 L'image permet d'opposer trois secteurs bien distincts sur l'archipel de Chausey: les surfaces en eau profonde de teinte homogène noire, les îles et les îlots végétalisés et dominés par des teintes rouge-orangées et l'estran qui présente, en liaison avec une granulométrie particulièrement variée (des faciès hétérogènes envasés aux sables très grossiers coquilliers), une gamme de teintes très diversifiées, témoignant d'une forte hétérogénéité spectrale, de caractéristiques physiques et paysagères de nature différente.

\section{Modalités de traitement des images}

Le traitement d'image a consisté en une série d'opérations supervisées réalisées sur les canaux de l'image SPOT5. La première phase a consisté à rechercher une nomenclature adaptée aux données images et au terrain. Dans un second temps, qui correspond à la phase d'étalonnage et d'échantillonnage, la connaissance du terrain a permis d'identifier et de générer sur les données images des zones de même attribution thématique. Enfin, une évaluation thématique a permis de valider les méthodes et les résultats des classifications en faisant intervenir les facteurs géographiques et écologiques du milieu dont il connaît l'influence sur la localisation et la dynamique des objets au sol (relief, marées, etc.). Ces contraintes du milieu sont également mises en relation avec celles liées à l'acquisition des données images et qui peuvent influer sur leurs qualités (heure de prise de vue, pré-traitements, etc.).

\section{Une nomenclature adaptée à la nature du terrain et à l'image}

Sur la base NATURA 2000, le Conservatoire du Littoral et des Espaces Lacustres, qui est l'opérateur local sur le site de l'archipel des îles Chausey, a déterminé 10 habitats génériques sur cette zone d'étude dont 3 qui correspondent au domaine de l'estran. Ces 3 habitats génériques sont les bancs de sable à faible couverture permanente d'eau marine (code 1110), les replats boueux ou sableux exondés à marée basse (code 1140) et les récifs (code 1170). Les deux thèmes 7 et 10 peuvent être considérés comme des indicateurs DCE (Directive Cadre Eau), de même que les classes « algues ». 
La nomenclature retenue ne considère que les surfaces en eau et les habitats de l'estran (tableau 1). Le choix définitif a été déterminé par une étape préalable qui correspond à une première lecture visuelle des images. Sur ces données sont recherchés, identifiés et retenus les postes de nomenclature qui présentent une bonne séparabilité spectrale. Cette étape fait appel à une bonne connaissance du terrain et à l'apport des photographies aériennes (fig. 4). Les postes de légende, associés à la zone intertidale, replats boueux, bancs sableux et récifs sont identifiables sur les données SPOT5. Mais la nomenclature retenue est également adaptée à la date et à l'horaire de prise de vue des images SPOT5. En effet, les images SPOT5 et les photographies aériennes n'ont pas été acquises aux mêmes dates ${ }^{2}$. Les niveaux d'eau sur l'archipel ne sont donc pas identiques : + $2 \mathrm{~m}$ SHOM environ lors du passage du satellite, $+4 \mathrm{~m}$ SHOM au passage du capteur aéroporté. Aussi, certains objets, détectables sur le premier document, ne le sont pas sur le second en raison de la hauteur d'eau.

Figure 4 : Quelques exemples d'objets benthiques relevés sur le terrain Some examples of benthic habitats on the field
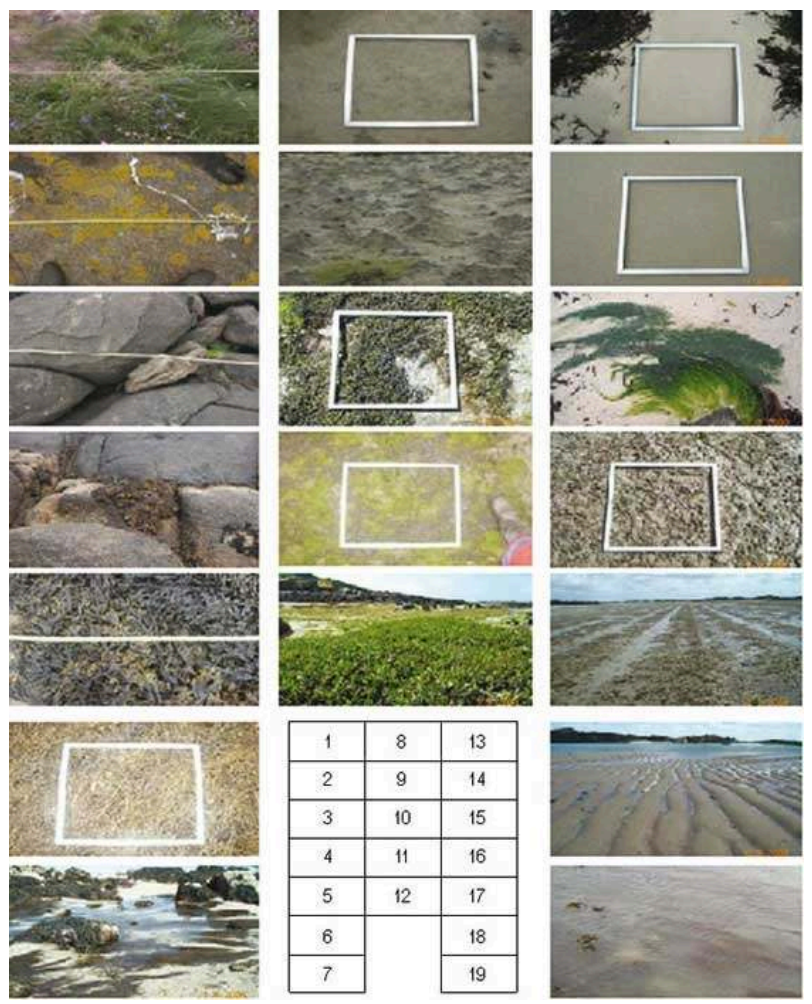

\begin{tabular}{|c|c|c|}
\hline 1 & 8 & 13 \\
\hline 2 & 9 & 14 \\
\hline 3 & 10 & 15 \\
\hline 4 & 11 & 16 \\
\hline 5 & 12 & 17 \\
\hline 6 & \multirow{3}{*}{} & 18 \\
\cline { 1 - 1 } 7 & \multirow{2}{*}{19} \\
\cline { 1 - 2 } & &
\end{tabular}

Substrats rocheux : 1. Végétation phanérogamique terrestre dans le supralittoral ; 2. Ceinture à lichens (Caloplaca marina) dans le supralittoral ; 3 . Ceinture à lichens (Verrucaria maura) dans le supralittoral inf./médiolittoral sup. ; 4. Ceinture de Pelvetia canaliculata et Lichina confinis dans le médiolittoral sup. ; 5. Ceinture de Fucus vesiculosuset F. spiralis dans le médiolittoral moy. ; 6. Ceinture d'Ascophyllum nodosum dans le médiolittoral moy. ; 7. Rochers recouverts d'A. nodosum et F. serratus et sables grossiers à Sargassum muticum dans le médiolittoral inf.

Substrats vaseux : 8 . Vase nue, non bioturbée, avec une pellicule de diatomées; 9 . Vase fortement bioturbée par Arenicola marina; présence d'Enteromorpha sp. ; 10. Vase recouverte par Fucus lutarius ; 11. Vase recouverte par F. lutarius, Enteromorpha sp. et Ulva sp. ; 12. Vase recouverte par une végétation de schorre (Salicornia sp. et Aster tripolium)

Substrats sableux : 13. Sable fin sec ressuyé avec épaves d'algues ; 14. Sable fin humide nu ; 15. Sable fin humide colonisé par Convoluta sp. (bloc rocheux avec Enteromorpha sp.) ; 16. Sable vaseux colonisé par Lanice conchilega; 17. Vénériculture (palourdes) et sillons colonisés par L. conchilega ; 18. Rides métriques dans les sables moyens; 19. Fond de chenal à sables grossiers hétérogènes colonisés par Sargassum muticum. 
Tableau 1: Correspondance des thèmes retenus pour la classification des données SPOT5 avec la nomenclature NATURA 2000

Linkage between SPOT5 data classified and Natura 2000 check list

\begin{tabular}{|c|c|c|}
\hline SPOTS & $\begin{array}{l}\text { Habitats generiques } \\
\text { NATURA } 2000\end{array}$ & $\begin{array}{l}\text { Habitats licmentairc } \\
\text { NATURA } 2000\end{array}$ \\
\hline 1. Eau profonde & & \\
\hline 2. Eau peu profonde & 1110 & $1110-1$ et $1110-2$ \\
\hline 3.Sable sec & 1140 & ${ }^{1140-1}$ \\
\hline 4. Sable humide & 1140 & $1140-3$ \\
\hline 5.Substrat vaso-sableux & 1140 & $1140-6$ \\
\hline 6. Substrat sablo-vaseux plus ou moins hum ide & 1140 & $1140-6$ \\
\hline 7. Banquettes à lanices & 1140 & $1140-6$ \\
\hline 8. Algues du bas médiolittoral & 1170 & $1170-2$ et $1170-3$ \\
\hline 9.Algues du haut médiolittoral & 1170 & $1170-2$ et $1170-3$ \\
\hline 10. Sargasses & 1110 & $1110-4$ \\
\hline 11. Lichens/rochers & 1170 & $1170-1$ \\
\hline 12. Rochers et algues & 1170 & $1170-9$ \\
\hline
\end{tabular}

La connaissance des signatures spectrales des objets est une étape importante qui permet de valider définitivement le choix des zones tests. Les missions terrain effectuées en mai et juillet 2003, mai et juillet 2004 ont par ailleurs permis d'améliorer considérablement l'échantillonnage de départ.

Les éléments relatifs aux caractéristiques propres des objets (densités d'individus par $\mathrm{m}^{2}$, étendue de leur occupation, etc.) et aux thèmes morpho-sédimentaires ont été observés sur le terrain. Ils expliquent les différences spectrales qui peuvent être rencontrées sur les images. Quatre familles de thèmes peuvent être inventoriés :

- Les substrats meubles sableux. Souvent hétérogènes, ces sédiments ont une granulométrie variable, des sables fins à grossiers, parfois bioclastiques. Ils sont localement bioturbés par les lanices ou recouverts par des populations de vers plats (Convoluta sp.) ;

- Les substrats meubles vaseux. Souvent humides, ces sédiments peuvent localement être très hétérogènes en mêlant des sables grossiers lithoclastiques aux vases. Ces vases peuvent aussi être bioturbées par les arénicoles (Arenicola marina) ou recouvert par des algues (Fucus lutarius, Vaucheria dichotoma var. marina. ou Enteromorpha spp.) ;

- Les substrats durs. Les rochers, très nombreux dans le secteur occidental, peuvent être nus ou recouverts par des lichens organisés en ceinture ;

- Les platiers rocheux. Sur ces platiers, les ceintures algales du médiolittoral se développent et s'organisent selon leur position sur l'estran (altitude) et selon le mode (orientation).

Ces relevés terrain fournissent une source incontournable (la seule) permettant d'analyser au mieux les radiométries enregistrées par l'image. Après les étapes du choix définitif de la nomenclature et celle des échantillons de classification, les polygones d'échantillons sont séparés de façon aléatoire en deux groupes distincts. Les polygones d'entraînement (servant au procédé de classification) forment un premier lot, le second lot correspond quand à lui aux polygones de validation (servant à vérifier la fiabilité de la méthode).

17 Au final, l'échantillonnage total représente $8,21 \%$ de la totalité des pixels de l'image, soit une très faible étendue de l'image traitée.

\section{Détermination du niveau de séparabilité spectrale des thèmes}

Les données SPOT5 utilisées se situent dans un contexte saisonnier relativement favorable à la reconnaissance des principaux thèmes recherchés. Cependant, il est à 
noter une forte ambiguïté entre les valeurs de la végétation terrestre essentiellement composée de landes et de prairies et les algues : à la date d'acquisition des données, ces deux classes thématiques présentent des comportements radiométriques très proches dans les trois bandes spectrales (fig. 5).

Figure 5 : Signature numérique des postes de nomenclature Spectral response of delected items

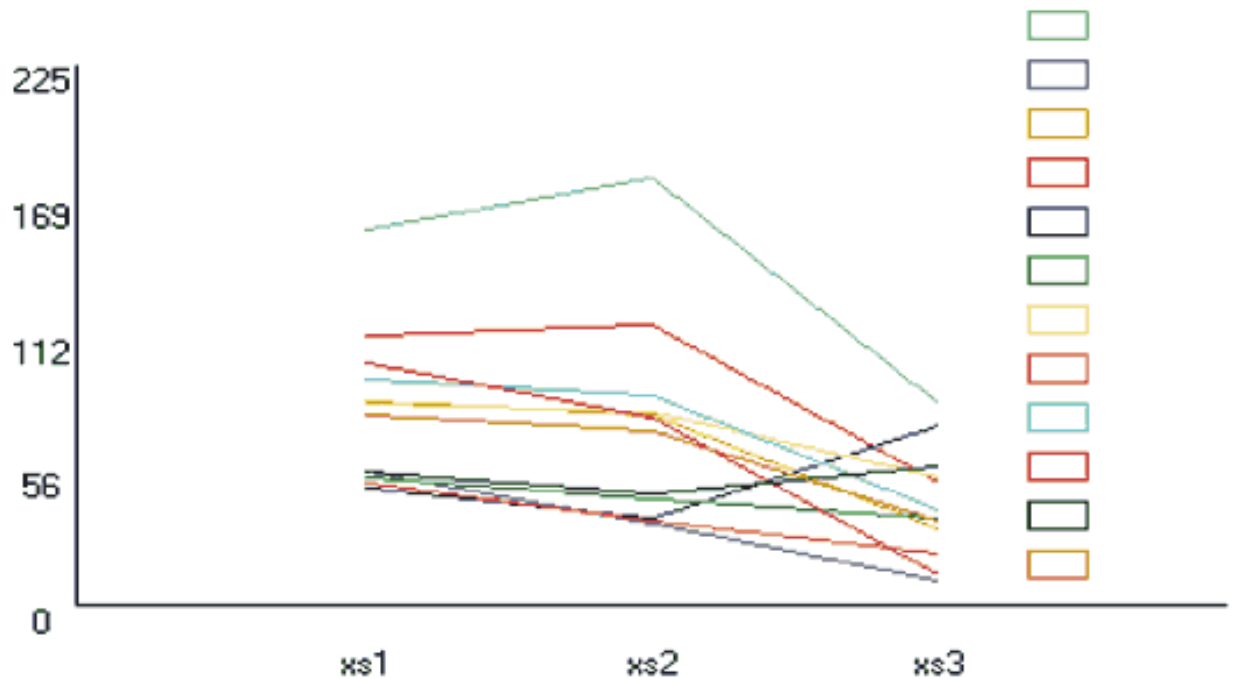

19 La méthode de classification appliquée dans le cadre de cette étude est une méthode supervisée, le maximum de vraisemblance, couramment utilisée en traitement d'images (fig. 6). La matrice de confusion compare les données classées aux données terrain, elle est utilisée pour analyser les résultats sur l'archipel (tableau 2). L'estimation globale de la classification est très correcte (coefficient de Kappa de l'ordre de 0,86 ) bien qu'il semble délicat de considérer la méthode sur le seul critère de sa performance générale.

Figure 6 : Classification par le maximum de vraisemblance des trois canaux XS1 XS2 XS3 (Image SPOT5 du 14 mai 2003)

Sea/land cover map derived from SPOT5 satellite data (14-03-2003)
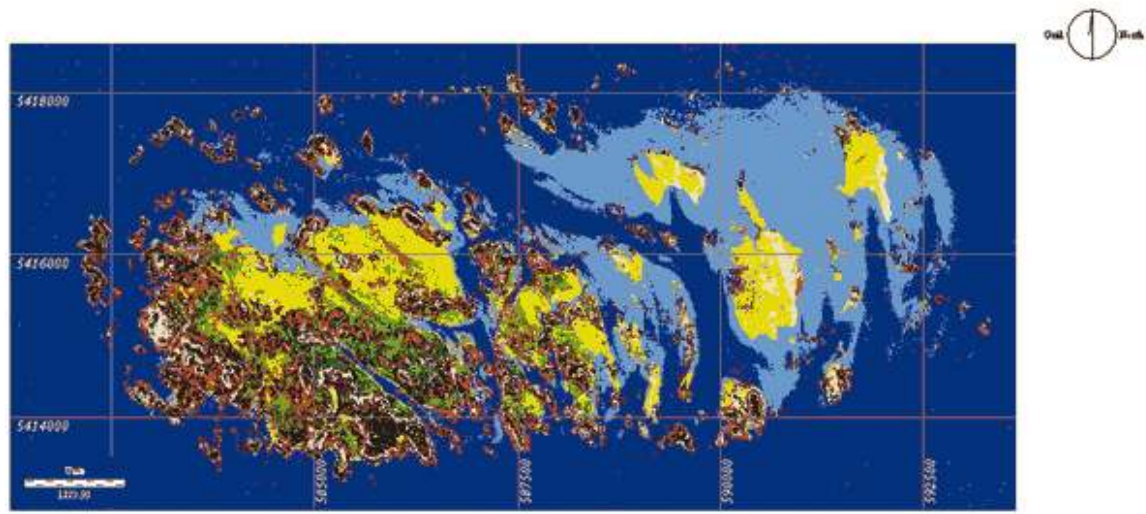
Tableau 2 : Matrice de contingence issue de classification par le Maximum de Vraisemblance Producer' accuracy, user's accuracy and overall accuracy for the classification image

\begin{tabular}{|c|c|c|c|c|c|c|c|c|c|c|c|c|c|c|}
\hline Thèmes & $\begin{array}{c}\text { Eal } \\
\text { profonde }\end{array}$ & $\begin{array}{l}\text { E2u peu } \\
\text { profonde }\end{array}$ & Sible & $\begin{array}{c}\text { Szble } \\
\text { humude }\end{array}$ & $\begin{array}{c}\text { Bunquettes } \\
\text { 2 hnikes }\end{array}$ & $\begin{array}{c}\text { Substrat } \\
\text { reso- } \\
\text { sibleix }\end{array}$ & $\begin{array}{l}\text { Substrat } \\
\text { s2blo- } \\
\text { raseux }\end{array}$ & \begin{tabular}{|l|} 
Algues du \\
bus mádo
\end{tabular} & $\begin{array}{l}\text { Algues du } \\
\text { hult málio }\end{array}$ & Sargases & $\begin{array}{l}\text { Lrchens } \\
\text { Rochers }\end{array}$ & $\begin{array}{l}\text { Rorhers } \\
\text { Algues }\end{array}$ & $\begin{array}{c}\text { Totzl } \\
\text { Com- } \\
\text { misstan }\end{array}$ & $\begin{array}{c}\text { Emeur } \\
\text { Eviu } \\
\text { profonde }\end{array}$ \\
\hline $\begin{array}{c}\text { E2u } \\
\text { profonde }\end{array}$ & 666848 & 0 & 0 & 0 & 0 & 0 & 0 & 0 & 0 & 0 & 0 & 0 & 666848 & $0, \infty 000$ \\
\hline $\begin{array}{l}\text { E2u peu } \\
\text { profonde }\end{array}$ & 417 & $6+2$ & 0 & 0 & 0 & 0 & 0 & 0 & 0 & 0 & 0 & 0 & 1059 & 0,3938 \\
\hline Sablesce & 0 & 0 & 800 & 44 & 0 & 0 & 0 & 0 & 0 & 0 & 0 & 0 & 844 & Q,0521 \\
\hline $\begin{array}{l}\text { Sable } \\
\text { humnde }\end{array}$ & 3 & 13 & 3 & 459 & 4 & 0 & 0 & 0 & 0 & 0 & 0 & 0 & 482 & 0,0477 \\
\hline $\begin{array}{l}\text { Banquettes } \\
\text { i hruces }\end{array}$ & 1 & 0 & 0 & 0 & 38 & 10 & 9 & 0 & 0 & 0 & 0 & 0 & 58 & 0,3448 \\
\hline \begin{tabular}{l|} 
Sulfritrit \\
rsso- \\
sableux
\end{tabular} & 0 & 0 & 0 & 0 & 67 & 145 & 1 & 0 & 0 & 0 & 0 & 0 & 213 & 0,3192 \\
\hline $\begin{array}{l}\text { Sulkstrit } \\
\text { sabb- } \\
\text { raseux }\end{array}$ & 0 & 0 & 0 & 0 & 94 & 0 & 119 & 0 & 0 & 0 & 0 & 0 & 213 & 0,4413 \\
\hline $\begin{array}{l}\text { Algues du } \\
\text { bus mádb }\end{array}$ & 0 & 0 & 0 & 0 & 0 & 0 & 0 & 368 & 0 & 0 & 0 & 18 & 386 & 0,0466 \\
\hline $\begin{array}{l}\text { Algues du } \\
\text { huut mâlb }\end{array}$ & 0 & 0 & 0 & 0 & 0 & 0 & 0 & 22 & 125 & 13 & 0 & 1 & 161 & 0,2236 \\
\hline Sangases & 277 & 0 & 0 & 0 & 0 & 0 & 0 & 0 & 10 & 337 & 0 & 0 & 624 & 0,459 \\
\hline $\begin{array}{l}\text { Lrthers } \\
\text { Rochers }\end{array}$ & 0 & 0 & 0 & 0 & 0 & 0 & 0 & 0 & 0 & 0 & 60 & 1 & 61 & 0,0164 \\
\hline $\begin{array}{l}\text { Rochers } \\
\text { Algues }\end{array}$ & 0 & 0 & 0 & 0 & 0 & 0 & 0 & 44 & 9 & 0 & 0 & 48 & 101 & 0,5248 \\
\hline Totzl & 667546 & 65 & 813 & 503 & 203 & 155 & 129 & 434 & 144 & 350 & 60 & 68 & 671050 & \\
\hline $\begin{array}{l}\text { Erreur } \\
\text { domisston }\end{array}$ & 0,010 & Q0198 & 0,0037 & Q085 & 0,8128 & 0,0645 & 0,0775 & 0,1521 & Q1319 & Q0371 & 0,000 & 0,2941 & & 0,0016 \\
\hline
\end{tabular}

Kappa $=0,8619$

En colonne : l'échantillonnage (vérité terrain) et les erreurs d'omission ; en ligne : la classification et les erreurs de commission; sur la diagonale : les pixels correctement classés

- Les classes « eau profonde », « lichens/rochers » et « sable sec » ne posent pas de problème particulier. Elles sont relativement bien classées sans erreur importante d'omission ou de commission. De légères confusions s'observent entre l'» eau peu profonde » et les « sargasses » (Sargassum muticum). Le « sable sec » est bien classé malgré une légère contamination vers la classe « sable humide».

- Les « banquettes à lanices » accusent des erreurs d'omission importantes $(0,81)$. La grande majorité des pixels échantillonnés en lanices a été attribuée à la classe « substrat sablovaseux », d'autres échantillons ont été affectés à la classe « substrat vaso-sableux » et, dans une moindre proportion, à la classe « sable humide ».

\section{Comparaison avec la photo-interprétation}

La cartographie par photo-interprétation des surfaces occupées sur l'archipel de Chausey apporte un état de référence qui peut être utilisé dans l'étape de validation du procédé de classification des images SPOT5 (Fournier et al., 2004). Le tableau 3 permet de comparer les résultats acquis en terme de surfaces et pour certains thèmes par traitement des données SPOT5 et photo-interprétation. 
Tableau 3 : Comparaison des surfaces SPOT 5 avec les surfaces relevées par photo-interprétation SPOT5 data classified compared to photographic areas

\begin{tabular}{|l|c|c|}
\hline Surfaces en ha & SPOT5 & $\begin{array}{c}\text { Photographie } \\
\text { aérienne }\end{array}$ \\
\hline Lanices & 139 & 140 \\
Algues (haut et bas médiolittoral) & 678 & 819 \\
Sargasses (S.muticum) & 119 & 22 \\
\hline
\end{tabular}

Surfaces en ha - photographie aérienne

\begin{tabular}{|l|l}
\hline Algues (haut et bas médiolittoral) & 819
\end{tabular}

Sargassum muticum $\quad 22$

Zostera marina $\quad 343$

Zostera noltii

Fucus lutarius

Vaucheria dichotoma var marina

Lanice conchilega

Schorre

2

2

12

140

3

Les différences entre les résultats obtenus par traitement d'image et ceux obtenus par photo-interprétation et levés terrain peuvent s'expliquer aisément :

- Dans le cas des lanices, la méthode de classification de l'image SPOT5 donne des résultats extrêmement proches de ceux obtenus par photographie aérienne et observations terrain.

- Le résultat obtenu sur Sargassum muticum est très différent. L'image SPOT5 a été acquise avec un niveau d'eau important. Par ailleurs, les sargasses, immergées au moment de la prise de vue, vivent à proximité des herbiers de phanérogames marines (Z. marina). C'est pourquoi SPOT5 a des difficultés à discriminer correctement les sargasses des zostères marines. Le résultat obtenu (119 hectares) montre cette confusion entre les sargasses ( 22 hectares en réalité) et les zostères marines (343 hectares). Les chiffres obtenus par SPOT5 ne sont pas non plus la somme des deux surfaces car une grande partie de l'herbier de phanérogame marine était nettement immergé et son signal se confondait avec celui de l'eau. Les données SPOT5, dans ce cas précis, montrent leurs limites.

- Enfin, les surfaces des algues donnent un résultat assez intéressant malgré la différence notable que l'ont peut observer, 678 hectares avec SPOT5, 819 hectares avec la photographie aérienne. La photographie aérienne permet de différencier les algues des platiers rocheux (fucales essentiellement) des autres algues (vasicoles précisément) et de la végétation phanérogamique de l'estran (zostère naine et schorre) ; ce que ne peut faire SPOT5. Il est donc plus correct de comparer la somme des surfaces couvertes par ces différents objets avec celles obtenues par SPOT. La différence s'explique, là encore, par la présence de l'eau. Certains secteurs immergés n'ont pu être détecté puisque le signal des algues recouvertes par l'eau se confondait avec celui de l'eau.

\section{Synthèse des résultats}

La classification a permis d'effectuer une cartographie quantitative des principaux thèmes de l'estran. Les résultats de la répartition spatiale des thèmes d'occupation du sol indiquent une forte dominance surfacique de la classe « eau profonde » suivie de 
l'» eau peu profonde ». Les «sables » occupent également une surface non négligeable de l'archipel. Les superficies recouvertes par les algues, près de 650 hectares, correspondent assez bien aux superficies relevées sur le terrain mais englobent également les surfaces végétalisées des îles. À cette période de l'année (mai), les stades végétatifs productifs des landes et prairies sont source de confusion avec les algues. Ces confusions s'expriment par des valeurs numériques moyennes très proches entre les deux thèmes dans les trois canaux du capteur. Ces problèmes liés à la date de prise de vue ne permettent pas d'estimer correctement les surfaces occupées par les algues. Les résultats des classifications font nettement apparaître la difficulté de mettre en évidence, avec un fort taux de réussite et de précision et à l'aide d'une seule date de prise de vue, l'ensemble des thèmes relatifs aux habitats benthiques de l'archipel des îles Chausey. La très faible étendue et le morcellement de certains des items ne permettent pas de les déceler malgré la résolution spatiale du capteur. Les banquettes à lanices (densité $>400$ individus $/ \mathrm{m}^{2}$ ) sont assez correctement évaluées avec la plupart des méthodes. Toutefois, il n'a pas été possible de détecter les secteurs où ces vers ont une faible densité. Elles se confondent alors avec les classes substrats qu'elles contribuent ainsi à surévaluer.

Certains points peuvent être observés :

- Sur les confusions spectrales : l'analyse des résultats issus de la classification montre qu'il existe un chevauchement important entre la végétation terrestre et les algues présentes sur l'estran. Sur ces images, il n'a pas été possible de les différencier. D'autre part, de fortes confusions et, par conséquent, des erreurs d'attribution finales apparaissent entre des surfaces qui sont :

- Différentes : l'eau est responsable de confusions radiométriques et de mauvais classement (entre les algues en général, mais aussi avec les phanérogames marines) ;

- Proches, voire identiques : les degrés d'humidité des sédiments, très variables, entraînent des omissions (substrat vaso-sableux, substrat sablo-vaseux) ;

- Identiques mais avec des recouvrements différents : densité variable des populations, présence de l'eau (lanices).

L'eau est donc le principal facteur de confusions entre les habitats benthiques

\section{Discussion}

\section{Des moyens et des techniques à améliorer}

Pour se limiter à la distinction entre les sédiments sableux ou vaseux et les surfaces couvertes ou colonisées (lanices, algues), la télédétection multispectrale à Très Haute Résolution Spatiale apporte des résultats satisfaisants, opérationnels et facilement reproductibles. Lorsqu'il s'agit d'accorder aux surfaces couvertes une affectation plus précise, la précision de l'inventaire, si elle est liée en partie à une date optimale de prise de vue et aux conditions du terrain, est également dépendante de la résolution spatiale $\mathrm{du}$ capteur. Par ses potentialités de résolution spatiale, SPOT5 améliore considérablement l'approche du milieu littoral. Toutefois, la distinction entre les différentes espèces d'algues, pourtant très différentes morphologiquement, reste très difficile. Ce constat peut sembler surprenant, cependant il est presque identique à celui que l'on peut faire en utilisant des photographies aériennes sur lesquelles a distinction 
entre les espèces est plus aisée mais il reste absolument indispensable d'effectuer un très lourd travail sur le terrain à des fins d'inventaires et de levés.

\section{La nomenclature}

26 La classification supervisée n'a pas permis de mettre en évidence tous les postes de nomenclature relevés par NATURA 2000. On remarquera que les objets de l'estran, identifiés ici par photo-interprétation ou par traitement de données satellitales, sont souvent difficilement "classables » dans les nombreuses nomenclatures mises à la disposition des thématiciens. Ceci s'explique d'une part parce que les références (bibliographiques) pour classer ces objets sont parfois différentes et d'autre part parce que ces objets, tels que l'on peut les identifier sur la photographie aérienne ou sur l'image satellite, ne se positionnent pas tous sur une même échelle biocénotique : c'està-dire qu'ils correspondent tantôt à des habitats (à un «biotope »), tantôt à un espace (notion de "zonation»), voire même parfois à un simple taxon (exemple des espèces ubiquistes comme Sargassum muticum). Une partie du problème tient à la nomenclature retenue (NATURA 2000) elle-même. On constate que 3 habitats génériques seulement couvrent l'ensemble de l'estran, ce qui constitue à Chausey près de 97,5\% de la superficie totale des espaces situés au-dessus du 0 marégraphique de l'archipel. Il en résulte que les surfaces couvertes par les autres "habitats» sont insuffisamment étendues pour être correctement cartographiées par SPOT5.

En outre, la typologie ne fait pas mention des termes «algues» et "phanérogames marines " alors qu'il existe un code (1120) pour l'herbier méditerranéen à Posidonies (Posidonia oceanica). Ces végétaux constituent toutefois un des intérêts majeurs de l'étude. Les herbiers de phanérogames marines de l'archipel des îles Chausey sont constitués principalement par Zostera marina et Zostera noltii. La surface couverte par ces végétaux est de 344 hectares en 2002 soit près de $12 \%$ de la surface de l'archipel (Fournier, 2003); résultat que l'on peut comparer à celui obtenu en 1982 soit 92 hectares ( $3 \%$ de la surface de l'archipel). Ces végétaux n'ont pu faire l'objet d'une cartographie en raison de la hauteur d'eau lors de l'acquisition de la scène SPOT5 étaient défavorables : les herbiers de phanérogames marines restant immergés.

Établir une typologie de ces éléments par télédétection relève donc souvent de l'appréciation de chaque auteur mais surtout des potentialités des données utilisées. Cela explique d'ailleurs les différences dans les nomenclatures utilisées en cartographies des littoraux par télédétection. Ces résultats montrent que pour cartographier ces objets avec des données satellitales de Très Haute Résolution, une catégorisation des postes de nomenclature demeure une étape obligée, dépendante des potentialités temporelle, spectrale et spatiale du capteur considéré mais aussi des conditions du milieu à la date d'acquisition des images.

\section{L'échantillonnage}

Les erreurs peuvent également être liées aux caractéristiques propres de l'objet : une classe peut n'être que très peu représentée à l'origine (lichens), ou présenter des réponses radiométriques spatialement variables (sable plus ou moins humide, sargasses plus ou moins couvertes d'eau); d'autres objets, bien que présents peuvent n'être identifiables qu'à partir d'une certaine densité de population (lanices). Ce type de classe 
nécessite un «dégroupage » préalable à la fusion des sous-classes, ainsi déterminées, dans une phase post-opératoire.

Si chaque objet doit être parfaitement identifié lors de l'échantillonnage, il arrive pourtant très souvent qu'il ne soit pas homogène dans sa constitution. Par exemple, les différentes ceintures algales (pourtant quasiment monospécifique) n'ont pu être isolées car il était impossible de dégager des échantillons propres à chaque espèce. C'est donc une ceinture plurispécifique qui a été discriminée. La mixité des réponses spectrales au sein d'un même thème se retrouve également pour la classe « banquettes à lanices ».

31 Enfin, la discrimination des radiométries des éléments du littoral n'a pu être effectuée en raison notamment d'intempéries lors des missions terrain de spectro-radiométrie. Avec l'utilisation d'un spectroradiomètre terrain, ces mesures auraient permis d'identifier précisément les signatures spectrales et les propriétés particulières des éléments du littoral (algues, sédiments, etc.) dans les domaines du Visible et du Proche Infrarouge.

Pour toutes ces raisons, s'il est possible de définir des échantillons fiables, il reste encore difficile de couvrir par échantillonnage l'ensemble des variations spectrales présentes sur les images. Quantifier la part respective de chacune des causes exposées reste un problème délicat.

\section{La prise en compte de l'environnement}

33 La localisation exacte des limites de surfaces occupées par les objets est parfois complexe et pour certains d'entre eux bien souvent imprécise. Ainsi, les banquettes à lanices sont aisément identifiables mais les surfaces occupées difficilement délimitables. Un gradient de densité, très irrégulier, apparaît du centre des zones colonisées par les lanices aux zones qui en sont dépourvues. Les méthodes fondées exclusivement sur des classifications radiométriques sont donc insuffisantes. Pour cette raison, il n'a été possible de cartographier que les secteurs présentant une densité supérieure à 400 individus $/ \mathrm{m}^{2}$. Ce résultat n'est pas étonnant : les lanices sont des vers polychètes qui construisent un tube fabriqué avec des grains de sable pour y vivre. La signature spectrale des lanices est donc extrêmement proche de celle du sable. Toutefois, les lanices modèlent leur environnement et structurent le milieu de telle façon que le sable colonisé par les lanices est presque toujours plus humide que le sable, situé au même niveau altimétrique, qui en est dépourvu. Le capteur détecte ainsi plus les contrastes sable humide/sable sec que les lanices proprement dites. Une solution consiste à détecter les changements de texture induits par des variations de densité au sol et par une localisation préférentielle des lanices dans les secteurs humides des chenaux. De la même façon, les confusions entre "algues du haut et bas médiolittoral » (Ascophyllum nodosum par exemple) avec les «rochers/algues» s'expliquent par le fait que ces dernières sont composées des précédentes!

L'intérêt des recherches à venir se situe donc dans la prise en compte de l'environnement de l'objet. Il faut en effet envisager des traitements permettant d'enrichir la représentation habituelle de texture fondée par la prise en compte des variations locales de contraste, par l'adjonction d'une connaissance des caractéristiques fixées par le terrain ou l'objet, par la prise en compte des possibilités de contacts entre les objets de l'estran (tableau 4). L'application des procédés de classification est susceptible de bénéficier directement de ces considérations qui consistent à prendre en 
compte les problèmes de la dimension, de l'organisation et de l'analyse de texture conjointement dans le domaine spatial et spectral.

\section{L'apport de données images acquises à une autre date}

Le passage systématique en matinée du satellite au-dessus de la zone d'étude ne permet pas d'obtenir des données de télédétection au niveau d'eau le plus faible à Chausey. Lors des grands coefficients de marée, la hauteur d'eau la plus basse est toujours l'après-midi. En outre, il serait très intéressant de compléter ces données par une prise de vue de l'archipel en période hivernale afin d'identifier les potentialités des données à des dates différentes, de détecter et de cartographier les changements intervenus sur l'estran. Les classifications multidates sont nécessaires pour mesurer les évolutions de l'état des surfaces, afin de différencier les changements spatiaux qui ont une signification écologique (ou climatique) de ceux qui traduisent un phénomène saisonnier lié aux cycles biologiques ou de marées. Dans l'idéal, il serait nécessaire de disposer d'une image, acquise à une date bien précise, par thème étudié : les herbiers de phanérogames marines en juin, les banquettes à lanices en juillet, certaines algues en mars, etc. Si la comparaison des classifications permet d'identifier les changements intervenus, le nombre des possibilités d'évolution peut être très important surtout quand le nombre de classes (thèmes) est élevé ou quand les changements sont liés aux phénomènes de déplacement de certains animaux ou végétaux (milieu dynamique).

Tableau 4 : Possibilités de contacts entre les objets de l'estran Potential contacts of macro tidal shore objets

\begin{tabular}{|c|c|c|c|c|c|c|c|c|c|c|c|c|}
\hline Pleine eau & Estrans & $\begin{array}{c}\text { Bancs } \\
\text { sableux } \\
\text { immergés }\end{array}$ & $\begin{array}{c}\text { Sable } \\
\text { sableux } \\
\text { immergés }\end{array}$ & $\begin{array}{c}\text { Banquettes } \\
\text { humide }\end{array}$ & $\begin{array}{l}\text { Sargasses } \\
\text { à Lanices }\end{array}$ & Vase & $\begin{array}{c}\text { Sable } \\
\text { humide }\end{array}$ & $\begin{array}{l}\text { Algues } \\
\text { émergé }\end{array}$ & $\begin{array}{c}\text { Algues } \\
\text { médio } \\
\text { sur blocs }\end{array}$ & $\begin{array}{l}\text { Pelvéties } \\
\text { médio }\end{array}$ & $\begin{array}{c}\text { Lichens } \\
\text { Lichens } \\
\text { noirs }\end{array}$ & clairs \\
\hline Pleine eau & & 0 & o & $\mathrm{x}$ & $\mathrm{X}$ & o & o & $\mathrm{X}$ & 0 & o & $\mathrm{x}$ & $\mathrm{X}$ \\
\hline $\begin{array}{l}\text { Estrans } \\
\text { sableux } \\
\text { immergés }\end{array}$ & o & & o & o & o & 0 & o & $\mathrm{x}$ & o & o & $\mathrm{x}$ & $\mathrm{X}$ \\
\hline $\begin{array}{l}\text { Bancs } \\
\text { sableux } \\
\text { immergés }\end{array}$ & o & o & & 0 & o & o & $\mathrm{x}$ & $\mathrm{x}$ & o & o & $\mathrm{x}$ & $\mathrm{x}$ \\
\hline $\begin{array}{l}\text { Sable } \\
\text { humide }\end{array}$ & $x$ & 0 & o & & 0 & 0 & o & 0 & o & o & o & $\mathrm{X}$ \\
\hline $\begin{array}{l}\text { Banquettes } \\
\text { à Lanices }\end{array}$ & $\mathrm{x}$ & 0 & o & o & & $\mathrm{x}$ & o & $\mathrm{x}$ & 0 & 0 & $\mathrm{x}$ & $\mathrm{x}$ \\
\hline Sargasses & o & 0 & 0 & 0 & $\mathrm{X}$ & & o & $\mathrm{X}$ & 0 & 0 & $\mathrm{X}$ & $\mathrm{X}$ \\
\hline $\begin{array}{l}\text { Vase } \\
\text { humide }\end{array}$ & o & o & $x$ & 0 & o & 0 & & o & 0 & o & o & $\mathrm{x}$ \\
\hline $\begin{array}{l}\text { Sable } \\
\text { émergé }\end{array}$ & $x$ & $x$ & $x$ & o & $x$ & $\mathrm{x}$ & o & & $\mathrm{x}$ & $x$ & $\mathrm{x}$ & $\mathrm{x}$ \\
\hline $\begin{array}{l}\text { Algues } \\
\text { médio sur } \\
\text { blocs }\end{array}$ & o & 0 & 0 & 0 & o & o & o & $\mathrm{x}$ & & o & o & $x$ \\
\hline $\begin{array}{l}\text { Algues } \\
\text { médio }\end{array}$ & o & o & o & o & o & o & o & $\mathrm{x}$ & o & & o & $\mathrm{x}$ \\
\hline $\begin{array}{l}\text { Pelvéties } \\
\text { Lichens } \\
\text { noirs }\end{array}$ & $\mathrm{x}$ & $\mathrm{x}$ & $\mathrm{x}$ & o & $\mathrm{x}$ & $\mathrm{x}$ & o & $x$ & o & o & & o \\
\hline $\begin{array}{l}\text { Lichens } \\
\text { clairs }\end{array}$ & $\mathrm{X}$ & $\mathrm{X}$ & $\mathrm{X}$ & $\mathrm{X}$ & $\mathrm{X}$ & $\mathrm{x}$ & $\mathrm{X}$ & $\mathrm{X}$ & $\mathrm{X}$ & $x$ & 0 & \\
\hline
\end{tabular}

\section{Conclusion}

La nécessité de préserver les habitats benthiques littoraux a été maintes fois démontrée. Les études concernant l'utilisation de la télédétection pour la cartographie et le suivi des changements des espaces littoraux sont de plus en plus fréquentes. De façon générale, l'étude des habitats benthiques est effectuée au travers de l'inventaire 
des éléments à des échelles fines, celle du terrain ou de la photographie aérienne. Les résultats obtenus avec les données satellitales soulèvent systématiquement le problème de la détection d'éléments dont la dimension au sol n'est pas en adéquation avec la résolution spatiale des capteurs. Ces travaux ne prennent généralement pas en compte les habitats élémentaires définis par la nomenclature NATURA 2000.

L'étude, menée sur l'archipel de Chausey, pose d'emblée le problème de la cartographie et du suivi des habitats naturels dans un cadre physique particulièrement contraignant. Il rappelle la nécessité de tenir compte des contraintes physiques du milieu littoral pour cartographier ses habitats les plus caractéristiques. Sur Chausey, le principal moteur de l'organisation spatiale des habitats est l'hydrodynamisme, qui est ici exceptionnel. Les objets de l'estran s'agencent selon leurs préférences écologiques en fonction de ces contraintes. C'est pourquoi, tout en utilisant une méthode classique pour l'inventaire des habitats benthiques littoraux, la démarche de ce travail s'est principalement orientée vers l'identification des potentialités des données de Très Haute Résolution Spatiale issues du capteur SPOT5. Les traitements ont été appliqués sur des images acquises au mois de mai et à un moment de fort coefficient marégraphique durant lequel l'estran présentait une grande étendue découverte. Ils ont permis d'identifier avec succès les principaux habitats benthiques de l'estran en prenant en compte les caractéristiques texturales des éléments paysagers du milieu. Ce travail a permis d'estimer les potentialités de ce capteur récent aux résolutions spatiales fines pour l'analyse des composantes du paysage littoral de Chausey. Il a également permis d'évaluer les limites des méthodes et du capteur lorsque les conditions locales ne permettent pas d'identifier les éléments du paysage. Les images du capteur SPOT5 ont un niveau de résolution spatiale relativement bien adapté sur des surfaces étendues et homogènes mais qui reste encore limité sur certaines catégories thématiques du littoral.

\section{BIBLIOGRAPHIE}

ANDRÉfouët (S.), CLAEREBOUdT (M.), MATSAKIS (P.), PAGÈs (J.), DUFOUR (P.), 2001. - « Typology of atolls rims in Tuamotu archipelago (French Polynesia) at landscape sclae using SPOT-HRV images ", International Journal of Remote Sensing, 22, p. 987-1004.

ANDRÉFOUËT (S.), KRAMER (P.), TORRES-PULIZA (D.), JOYCE (K. E.), HOCHBERG (E. J.), GARZA-PEREZ (R.), 2003. - « Multi-site evaluation of IKONOS data for classification of tropical coral reef environments ", Remote Sensing of Environment, 88, p. 123-143.

BELSHER (T.), 1985. - « Télédétection des végétaux marins du phytobenthos du littoral français : l'archipel des îles Chausey », Photo-Interprétation, 1985-5, 1, p. 1-7.

-, 1990. - « Apport du satellite SPOT à la cartographie des végétaux marins », Bulletin de l'Institut Océanographique de Monaco, spécial 6, p. 61-69. 
BENMOUSSA (H.), VIOLLIER (M.), BELSHER (T.), 1989. - « Télédétection des algues macrophytes de l'Archipel de Molène (France). Radiométrie de terrain et application aux données du satellite SPOT », International Journal of Remote Sensing, 10, 1, p. 53-69.

BONNOT-COURTOIS (C.), CALINE (B.), L'HOMER (A.), LEVOT (M.), 2002. - La Baie du Mont-Saint-Michel et l'estuaire de la Rance, Mémoire 26, TOTAL/FINA/ELF, CNRS, EPHE, Pau, 256 p.

BONNOT-COURTOIS (C.), ROLLET (C.), FOURNiER (J.), POPUlus (J.), GUILLAUMONT (B.), LOARER (R.), (sous presse). - « Cartographie bio-morpho-sédimentaire des estrans dans le cadre du réseau benthique. Complémentarité des orthophotographies littorales et des données Lidar », PhotoInterprétation.

CHAUVAUd (S.), BOUCHON (C.), MANIÈRE (R.), 2001. - « Cartographie des biocénoses marines de Guadeloupe à partir de données SPOT (récifs coralliens, phanérogames marines, mangroves) », Oceanologica Acta, 24, 1, p. 3-16.

DiersSen (H. M.), ZimMerman (R. C.), LeATHers (R. A.), DOWNes (T. V.), DAVis (C. O.), 2003. - « Ocean color remote sensing of seagrass and bathymetry in the Bahamas Banks by high-resolution airborne imagery ", Limnology and Oceanography, 48, 1, part 2, p. 444-455.

DRÉAU (A.), 2003. - Contribution de la télédétection aéroportée à la cartographie de l'estran. Application à l'archipel des Chausey, mémoire de DEA de Géographie, Université Rennes 2, 75 p.

FOURNIER (J.), 2003. - Cartographie des herbiers de phanérogames marines de l'archipel des îles Chausey, rapport DIREN Basse-Normandie, Dinard, $21 \mathrm{p}$.

fournier (J.), COTONNeC (A.), ANSelme (B.), GOUery (P.), TAlec (P.), PANizza (A. C.), MOKRANi (M.), DRÉAu (A.), LEVOT (M.), 2004. - Premières évaluations SPOT5 pour la cartographie des habitats benthiques littoraux de l'Archipel des Chausey (Manche), rapport final CNES, Toulouse, $94 \mathrm{p}$.

FOURNIER (J.), LEVOT (M.), 2004. - Carte des habitats benthiques médiolittoraux de l'archipel des Chausey (Manche), Laboratoire de Géomorphologie et Environnement littoral EPHE/CNRS, Dinard.

GUILLAUMONT (B.), BAJJOUK (Т.), TALEC (Р.), 1997. - « Seaweed and remote sensing : a critical review of sensors and data processing ", Progress in Phycological Research, 12, p. 213-282.

GUILLAUMONT (B.), CALLENS (L.), DION (P.), 1993. - « Spatial distribution and quantification of Fucus sp. and Ascophyllum nodosum beds in intertidal zones using SPOT imagery », Hydrobiologia, 260/261, p. 297-305.

LOUBERSAC (L.), BELBEOCH (G.), 1984. - « Haute resolution spatiale et environnement littoral (simulation SPOT, Bretagne, France) », Photo-Interprétation, 1984-5, 3, p. 19-23.

MEINESZ (A.), BELSHER (T.), BOUdOURESQUe (C.-F.), LEFEVRE (J.-R.), 1991. - « Première évaluation des potentialités du satellite Spot pour la cartographie des peuplements benthiques superficiels de Méditerranée occidentale ", Oceanologica Acta, 14, 3, p. 299-307.

MOKRANI (M.), 2004. - Évaluation des potentialités SPOT5 pour la cartographie des habitats benthiques médiolittoraux de l'archipel des Chausey, DEA de Géographie, Université Rennes 2, 91 p.

MUMBY (P. J.), EDWARDS (A. J.), 2002. - « Mapping marine environments with IKONOS imagery : enhanced spatial resolution can deliver greater thematic accuracy ", Remote Sensing of Environment, 82, p. 248-257.

PASQUALINI (V.), PERGENT-MARTINI (C.), FERNANDEZ (C.), PERGENT (G.), 1997. - « The use of airbone remote sensing for benthic cartography : advantages and reliability ", International Journal of Remote Sensing, 18, 5, p. 1167-1177. 


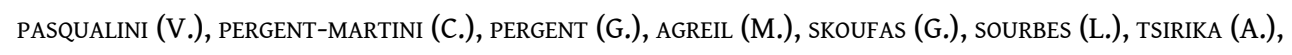
2005. - « Use of SPOT5 for mapping seagrasses : An application to Posidonia oceanica », Remote Sensing of Environment, 94, p. 39-45.

POPUlus (J.), LAURENTiN (A.), ROLLET (C.), VASQUez (M.), GUILLAUMONT (B.), BONNOT-COURTOIS (C.), 2004. "Surveying coastal zone topography with airborne remote sensing for benthos mapping ", Journal of Remote Sensing and Photogrammetry, 3, 1, p. 105-117.

SHANMUGAM (S.), LUCAS (N.), PHIPPS (P.), RICHARDS (A.), BARNSLEy (M.), 2003. - « Assessment of Remote Sensing Techniques for habitat mapping in coastal dune ecosystems ", Journal of Coastal Research, 19, 1, p. 64-75.

\section{NOTES}

1. DIREN Basse-Normandie, Conservatoire du Littoral, SCI de Chausey.

2. Mission aérienne : DIREN/IGN FR 5539/100 C du 13 août 2002, heure : 16 h 13-16 h 33, marée : Saint-Malo UT +1 : 10 h $2312 \mathrm{~m}$ - 17 h 14 1,65 m ; Mission SPOT5 : 14 mai 2003, heure : $11 \mathrm{~h} 33$, marée : Saint-Malo UT +1 : 05 h 25 11,50 m - 12 h 23 1,85 m. Corrections sur Chausey (Grande-île) : heures au port principal de référence (Saint-Malo) PMVE $+0 \mathrm{~h} 05 \mathrm{BMVE}+0 \mathrm{~h} 15$; hauteurs au port principal de référence (Saint-Malo) PMVE +0,8 $\mathrm{m}$ BMVE +0,45 $\mathrm{m}$.

\section{RÉSUMÉS}

Cette étude présente l'intérêt et les limites des données de télédétection à Haute Résolution Spatiale issues du capteur SPOT5 pour la cartographie des habitats benthiques médiolittoraux. Le site d'étude, l'archipel des îles Chausey (golfe normand-breton, France) se caractérise par un très vaste estran parsemé d'une multitude d'îlots. Par ses contraintes physiques, il constitue un site de recherche privilégié pour répondre aux problèmes actuels de cartographies des habitats littoraux, par ailleurs en cours de réalisation (programme REBENT-IFREMER) et initiées par les instances publiques (services délocalisés de l'État) suite aux nombreuses pollutions côtières. Des méthodes de traitement d'images traditionnelles et couramment utilisées ont été testées et validées à l'échelle de l'archipel par des photographies aériennes récentes et des campagnes d'échantillonnages faites sur le terrain. La classification retenue est très proche de celle de la nomenclature NATURA 2000 en ce qui concerne les habitats naturels de l'estran. Les traitements apportés permettent d'explorer quelques potentialités de SPOT5 et d'afficher ainsi une première évaluation de ces données pour la caractérisation, l'inventaire et le suivi des habitats naturels littoraux.

Recent coastal pollutions have induced local and national authorities (research institutes IFREMER, CNES, IFEN -, decentralised state agencies) to evaluate present mapping techniques and to assess their ability to map coastal benthic habitats. Within these research programs a test site has been choosen to evaluate data from the High Spatial Resolution Satelite SPOT5. It is the Chausey archipelago, in the English Channel which is characterized by a very wide macro tidal shore line with many skerries and very small islands. Such a rugged inter-and sub-tidal topography makes it a very demanding site for cartography. SPOT5 data has been classified 
(using usual routines of image processing), compared to photos, cross checked with field evidences. This has allowed to point out SPOT5 potential : the classification is very similar to the Natura 2000 check list and shows a good ability to map this type of shore. This preliminary test is a begining to a wider program of coastal monitoring by SPOT5.

\section{INDEX}

Index géographique : France, Chausey (île des)

Keywords : inhabiting, SPOT5

Mots-clés : habiter, SPOT5

\section{AUTEURS}

\section{ADELINE COTONNEC}

COSTEL - UMR 6554 CNRS LETG, Université Rennes 2 Haute Bretagne, Maison de la Recherche en Sciences Sociales, Place du Recteur Henri Le Moal - 35043 Rennes cedex, France, adeline.cotonnec@uhb.fr

\section{PASCAL GOUÉRY}

COSTEL - UMR 6554 CNRS LETG, Université Rennes 2 Haute Bretagne, Maison de la Recherche en Sciences Sociales, Place du Recteur Henri Le Moal - 35043 Rennes cedex, France, pascal.gouery@uhb.fr

\section{MOUNA MOKRANI}

COSTEL - UMR 6554 CNRS LETG, Université Rennes 2 Haute Bretagne, Maison de la Recherche en Sciences Sociales, Place du Recteur Henri Le Moal - 35043 Rennes cedex, France, mouna.mokrani@uhb.fr

\section{JÉRÔME FOURNIER}

Géomorphologie et Environnement littoral, UMR 8586 PRODIG CNRS, École pratique des hautes études, 2 rue Valette - 75005 Paris, France, jeromefournier@wanadoo.fr

\section{BRICE ANSELME}

UMR 8586 CNRS PRODIG, Université Paris 1 Panthéon-Sorbonne, 2 rue Valette - 75005 Paris, France,

banselme@univ-paris1.fr

\section{ALAIN DRÉAU}

COSTEL - UMR 6554 CNRS LETG, Université Rennes 2 Haute-Bretagne, Maison de la Recherche en Sciences Sociales, Place du Recteur Henri Le Moal - 35043 Rennes cedex, France.

Géomorphologie et Environnement littoral, UMR 8586 PRODIG CNRS, École pratique des hautes études, 2 rue Valette - 75005 Paris, France. 


\section{VINCENT DUBREUIL}

COSTEL - UMR 6554 CNRS LETG, Université Rennes 2 Haute Bretagne, Maison de la Recherche en Sciences Sociales, Place du Recteur Henri Le Moal - 35043 Rennes cedex, France,

vincent.dubreuil@uhb.fr

\section{ANDREA C. PANIZZA}

COSTEL - UMR 6554 CNRS LETG, Université Rennes 2 Haute-Bretagne, Maison de la Recherche en Sciences Sociales, Place du Recteur Henri Le Moal - 35043 Rennes cedex, France.

Géomorphologie et Environnement littoral, UMR 8586 PRODIG CNRS, École pratique des hautes études, 2 rue Valette - 75005 Paris, France.

\section{PASCAL TALEC}

DIREN Basse-Normandie, Avenue de Tsukuba, 14209 Hérouville-Saint-Clair Cedex, France, pascal.talec@basse-normadie.eclogie.gouv.fr 